\title{
RheoDSC: A hyphenated technique for the simultaneous measurement of calorimetric and rheological evolutions
}

\author{
S. Kiewiet \\ Department of Materials and Chemistry-Physical Chemistry and Polymer Science, \\ Vrije Universiteit Brussel, Pleinlaan 2, 1050 Brussels, Belgium \\ V. Janssens \\ Department of Chemical Engineering, Applied Rheology and Polymer Processing, \\ Katholieke Universiteit Leuven, W. de Croylaan 46, 3001 Leuven, Belgium \\ H. E. Miltner and G. Van Assche \\ Department of Materials and Chemistry-Physical Chemistry and Polymer Science, \\ Vrije Universiteit Brussel, Pleinlaan 2, 1050 Brussels, Belgium \\ P. Van Puyvelde \\ Department of Chemical Engineering, Applied Rheology and Polymer Processing, \\ Katholieke Universiteit Leuven, W. de Croylaan 46, 3001 Leuven, Belgium \\ B. Van Mele \\ Department of Materials and Chemistry-Physical Chemistry and Polymer Science, \\ Vrije Universiteit Brussel, Pleinlaan 2, 1050 Brussels, Belgium
}

(Received 31 July 2007; accepted 3 January 2008; published online 19 February 2008)

A newly developed hyphenated technique is presented combining an existing rheometer and differential scanning calorimeter into a single experimental setup. Through the development of a fixation accessory for differential scanning calorimeter (DSC) crucibles and a novel rotor, the simultaneous measurement is performed inside the well-controlled thermal environment of a Tzero $^{\mathrm{TM}}$ DSC cell. Hence, the evolution of thermal and flow properties of a material can be simultaneously measured using steady or oscillatory shear measurements and regular or modulated temperature DSC measurements. Along with the construction of a prototype, a validation of the design was performed. The technique offers interesting opportunities for the investigation of flow-induced transitions, for instance, crystallization or phase separation, and provides an asset for high-throughput screening of materials. The potential of the novel technique is demonstrated by two case studies: the chemorheology during the cure of a thermosetting epoxy-amine system and the flow-induced crystallization of syndiotactic polypropylene. (C) 2008 American Institute of Physics. [DOI: $10.1063 / 1.2838585]$

\section{INTRODUCTION}

To ensure proper processing of a material, the knowledge about its flow behavior and thermal properties is of crucial importance. The state-of-the-art approach for investigating these parameters requires the use of several analytical techniques, since a single technique does not provide sufficient understanding of the complex material behavior. For instance, transition temperatures are typically measured by means of a (modulated temperature) differential scanning calorimeter [(MT)DSC], whereas flow properties are commonly obtained from rheological testing. However, an accurate comparison of the quantities measured on separate instruments-for instance, viscosity and loss angle versus heat content and heat capacity-is not straightforward, since the measuring conditions-such as sample size, sample morphology, and temperature control-are often quite different. Moreover, an additional problem arises from the fact that different samples, not necessarily exhibiting the same kinetics, have to be measured in different instruments. Especially if one aims at investigating (flow-induced) material transfor- mations that are associated with changes in rheological and thermal properties, such as polymerization, melting and crystallization, or phase separation and remixing, an accurate comparison between the techniques is required. Shearinduced crystallization can be studied with in situ synchrotron small-angle $\mathrm{x}$-ray scattering and wide-angle $\mathrm{x}$-ray diffraction after a step shear under isothermal conditions, ${ }^{1,2}$ however, these techniques do not allow for a simultaneous rheological measurement and are not suitable for following a polymerization.

In this work, a hyphenated technique is developed that allows for a combined measurement of rheological and calorimetric properties. A similar concept was put forward by Martins et al., ${ }^{3}$ Rheometric Scientific (currently TA Instruments), ${ }^{4}$ and Nagatake et al. ${ }^{5}$ However, the drawbacks associated with their approaches limit the applicability of the instruments. Martins et $a l^{3}$ developed a shear differential thermal analyzer that allowed studying the effect of controlled shear pulses on the isothermal and nonisothermal solidifications of polymers. For this purpose, a capillary rheometer and a differential thermal analyzer were combined 
into a single instrument. The setup basically consists of two capillaries, one for the investigated polymer and another for a reference material, which are located in separated but identical ovens. A major drawback of the system is the choice of the reference material: it should have a heat capacity and thermal conductivity similar to that of the polymer under investigation, something which is very difficult to achieve when studying unknown samples. Secondly, due to the relatively large sample volume required, important temperature gradients develop across the cross section of the sample, leading to temperature lags during nonisothermal measurements. The approach described by Rheometric Scientific and Nagatake et al., ${ }^{4,5}$ consists of a commercial plate-plate rheometer with a modified lower plate containing two thermocouples, one positioned closely underneath the test sample and one mounted in the base plate, and as such allowing for the measurement of temperature differences resulting from sample transformations, as in a differential thermal analyzer. Since the temperature difference is only detected at a single axial distance, the technique only provides a local measurement of the heat flow and does not necessarily represent the overall response of the sample. Moreover, since the upper plate is not directly heated or cooled, vertical temperature gradients are likely to occur in view of the generally low thermal conductivity of the test specimen. Finally, since the thermocouples are in different arrangements, the temperature difference also contains contributions from the instrumental setup in addition to those related to the sample's response. Such difficulties could be overcome by calibration procedures, which are, however, not documented in the patent. ${ }^{4}$

To overcome the drawbacks associated with previous instrument designs, a commercial DSC cell was modified to accommodate the rotor connected to a commercial rheometer. By measuring directly in the DSC cell, one assures a quantitative temperature control. As a result, steady shear or small angle oscillatory shear rheometry measurements can be combined with linear or MTDSC measurements; the latter enabling the simultaneous measurement of heat flow and heat capacity. ${ }^{6,7}$ First, the design of the new assemblies will be discussed. Subsequently, the rheological and calorimetric signals are analyzed separately, as to provide the necessary validation of the device. To conclude, the potential of the technique is highlighted by two case studies.

\section{DESIGN AND CONSTRUCTION OF THE INSTRUMENT}

A prototype RheoDSC instrument was constructed starting from two stand-alone instruments: a Q1000 Tzero DSC (TA Instruments) and an AR1000 rheometer (TA Instruments). The Q1000 Tzero DSC uses the Tzero ${ }^{\mathrm{TM}}$ technology, ${ }^{8,9}$ which is of importance in view of the modifications that will be introduced into the cell when upgrading to a RheoDSC setup. The Tzero thermocouple, located between the sample and reference sensors, allows for a detailed mapping of the thermal environment of the sample and allows accounting for the thermal resistances and capacitances of the various heat flow paths in the cell.

To be able to conduct a rheological measurement inside a DSC crucible located on a DSC sensor, important changes

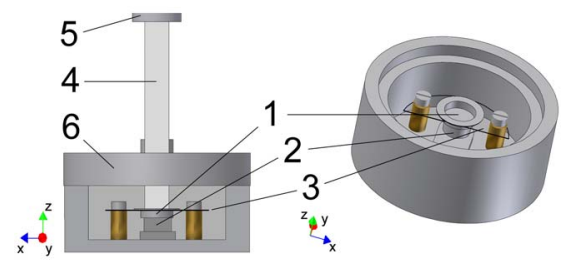

FIG. 1. (Color online) Principle of the RheoDSC setup for rheological measurements on a sample in a DSC crucible inside a DSC-cell: (left) overview of RheoDSC cell and (right) detailed view of the fixation of the DSC crucible (1) on the Tzero sensor (2) using a mica film (3). Other parts: IPC rotor (4), coupling part (5), silver lids (6). Left picture is view along $Y$-axis.

were made with respect to the traditional rheometer configuration (see Fig. 1). In a conventional rheometer, geometries often have a diameter in the order of $20 \mathrm{~mm}$ or more. Since the rheological measurement will be performed inside the DSC crucible, with a typical inner diameter of merely $5 \mathrm{~mm}$, a new rotor was developed with a diameter of $4.5 \mathrm{~mm}$. It consists of two parts: one part in contact with the sample, being used for imposing the necessary shear condition, and a second part designed to connect the former with the rheometer spindle. In this manner, a parallel plate geometry is achieved with the DSC crucible as the stator. To minimize the influence of the required modifications to the DSC cell, any new heat flow path introduced in the cell should possess a sufficiently high thermal resistance. Thus, only rotor materials with a low thermal conductivity and a low thermal expansion coefficient are suitable. In the current setup, an inorganic phosphate cement ${ }^{10}$ (IPC) has been selected. The coupling part, connecting the IPC rod to the rheometer spindle, is made of aluminum, mainly because of its easy shaping. The thermal resistance of this part is not an issue since it is not in direct contact with the sample, as can be seen in Fig. 1.

On the one hand, the DSC crucible acts as the stator of the rheological setup; on the other hand, it establishes the thermal contact between the sample and the DSC sensor. Hence, a reliable, motion-free positioning of the crucible onto the DSC sensor is required. An appropriate fixation accessory has been developed and is sketched in Fig. 1. The DSC crucible is attached to a thin supporting film that is fixed into the DSC cell by screw fitting onto cylindrical holders on both sides of the sensors. As the film is in direct thermal contact with the crucible and located inside the DSC cell, this material should possess a high thermal resistance in order to limit heat losses. Furthermore, high requirements are imposed on the mechanical resistance of the film, since it should be capable of withstanding the mechanical stresses imposed by the oscillation or steady rotation of the rotor. In this respect mica was selected because of its excellent thermal and mechanical characteristics in a broad temperature range.

To minimize external noise on the calorimetric measurements, a proper thermal shielding of the DSC cell is needed. For this purpose the standard Q1000 DSC cell configuration has been employed, with the DSC oven covered by two silver lids and an additional heat shield (Fig. 2). To position the 


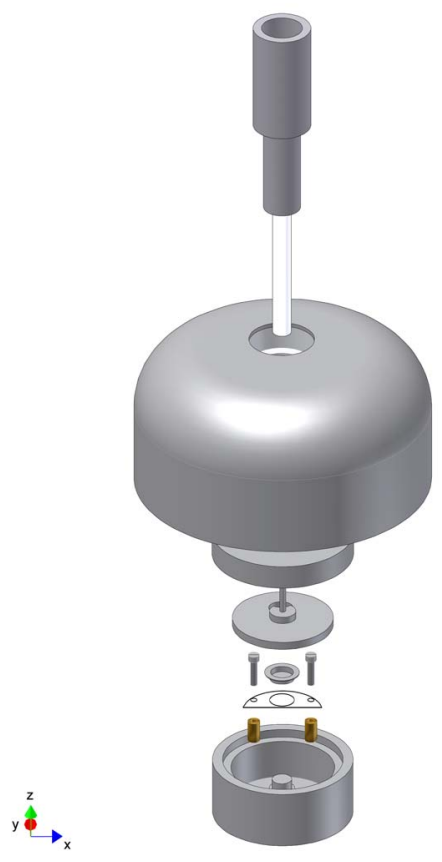

FIG. 2. (Color online) Exploded view of the RheoDSC setup showing the mounting of the mica film, the thermal shielding of the DSC cell by two silver lids and a heat shield, and the insertion of the IPC rotor with its coupling part.

silver lids in a reproducible manner onto the cell and to prevent obstruction of the rotor by these lids, a three point positioning system was mounted on top of the cell. The heat shield used is the conventional Q1000 heat shield that consists of a thermally insulating, porous ceramic material surrounded by a metal shell. In the silver lids and the heat shield, holes through which the rheometer geometry can be lowered were drilled. Care was taken to design the holes as small as possible to maintain the shielding quality.

\section{VALIDATION OF THE RheoDSC}

First, the validation of the rheological signals is discussed. The use of a $4.5 \mathrm{~mm}$ geometry as the rotor and a fixed DSC crucible as the stator constitutes a major modification that might have considerable implications for the quality of the rheological signal. As a test case, linear viscoelastic measurements obtained with a regular rheometer setup and with the RheoDSC setup will be compared.

As a first reference material, a calibration oil of known viscosity (Brookfield oil, $1000 \mathrm{~Pa} \mathrm{~s}$ at $25^{\circ} \mathrm{C}$ ) was used. Figure 3(a) shows the viscoelastic properties as a function of frequency at $25^{\circ} \mathrm{C}$ obtained with the $4.5 \mathrm{~mm}$ IPC geometry (filled symbols) and with a conventional rheometer setup using a parallel plate geometry (open symbols). The results are very similar over a broad frequency range. Only at high frequencies, small deviations in the rheological signals are noticed. The latter is not unexpected since inertia will become more of a concern at high frequencies in the case of a smaller geometry.

As a second reference material, a uncross-linked poly(dimethylsiloxane) (PDMS) (provided by TA Instruments)
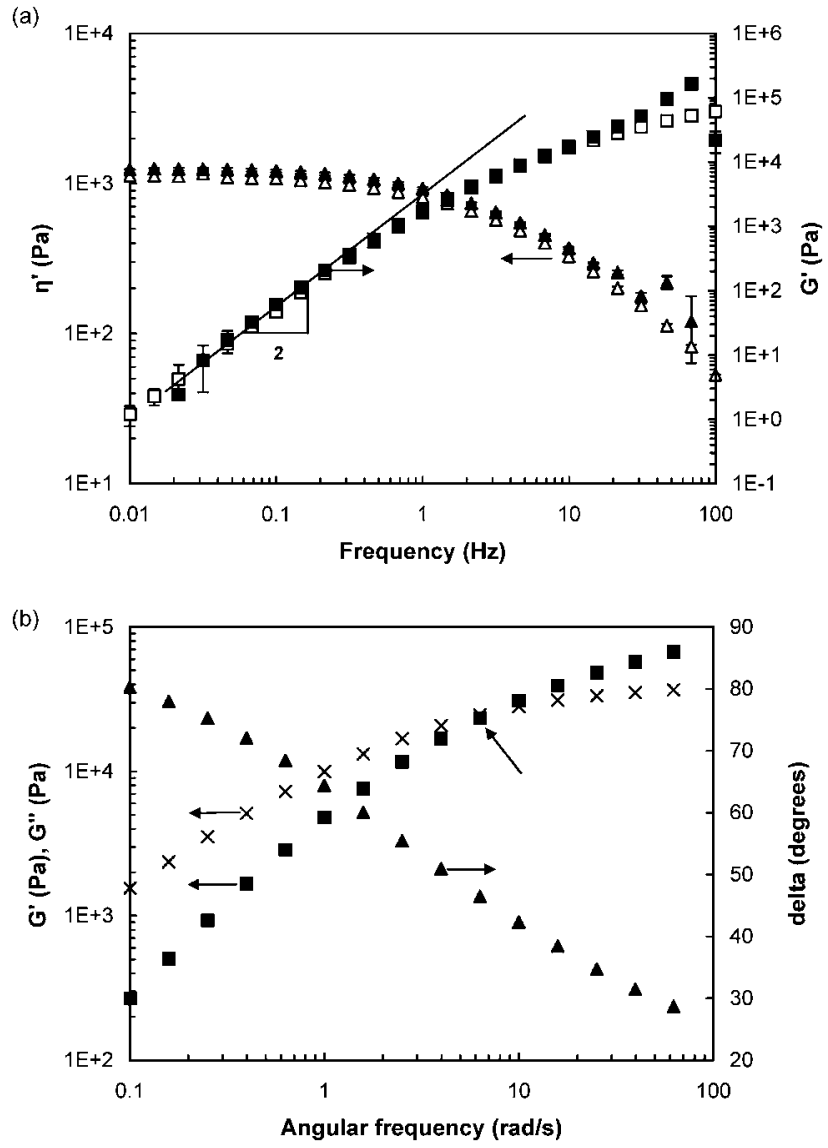

FIG. 3. Validation of the rheological signals: (a) Storage modulus ( $\square$ ) and dynamic viscosity $(\triangle)$ for a frequency sweep (strain $\gamma=0.05$ ) on a calibration oil with a viscosity of $1000 \mathrm{Pas}$ at $25^{\circ} \mathrm{C}$ using a $2.5 \mathrm{~cm}$ parallel plate (open symbols) and the $4.5 \mathrm{~mm}$ IPC rotor of the RheoDSC (filled symbols). Standard deviation on three experiments indicated. (b) Storage modulus (ם), loss modulus $(\times)$, and phase angle $(\mathbf{\Lambda})$ for a frequency sweep (strain $\gamma=0.02)$ at $25^{\circ} \mathrm{C}$ on a PDMS reference material using the RheoDSC setup.

was used. The experimental result obtained with the RheoDSC setup is given in Fig. 3(b). As in a regular rheometer, the elastic character of the viscoelastic material increases with increasing frequency, leading to a crossover of $G^{\prime}$ and $G^{\prime \prime}$ at $\omega_{c}$ equal to $7.4 \pm 0.3 \mathrm{rad} \mathrm{s}^{-1}$ and $G_{c}$ equal to $25.8 \pm 0.3 \mathrm{kPa}$ (based on three measurements). Measured with a regular rheometer, the crossover occurs at $\omega_{c}$ equal to $5.75 \pm 0.29 \mathrm{rad} \mathrm{s}^{-1}$ and $G_{c}$ equal to $25.5 \pm 2.0 \mathrm{kPa}$. Although the crossover frequency is somewhat higher in the RheoDSC setup, the modulus at crossover is very comparable. Overall, the rheological measurements are of sufficient quality, notwithstanding the small rotor and the DSC crucible stator.

In the course of the validation of the calorimetric signals, different configurations of the DSC cell were explored. As a general requirement, a more symmetric modification of the cell proved essential to prevent a severe deterioration of the heat flow signals for both first and pseudo-second-order transitions. Moreover, to obtain a stable heat flow signal during isothermal tests, the silver lids and heat shield proved to be of major importance. Finally, to ensure a more uniform temperature distribution inside the cell, a flow of inert helium purge gas was required. In the final setup, the isothermal baseline shows variations of about $5 \mu \mathrm{W}$ over $10 \mathrm{~min}$, which 

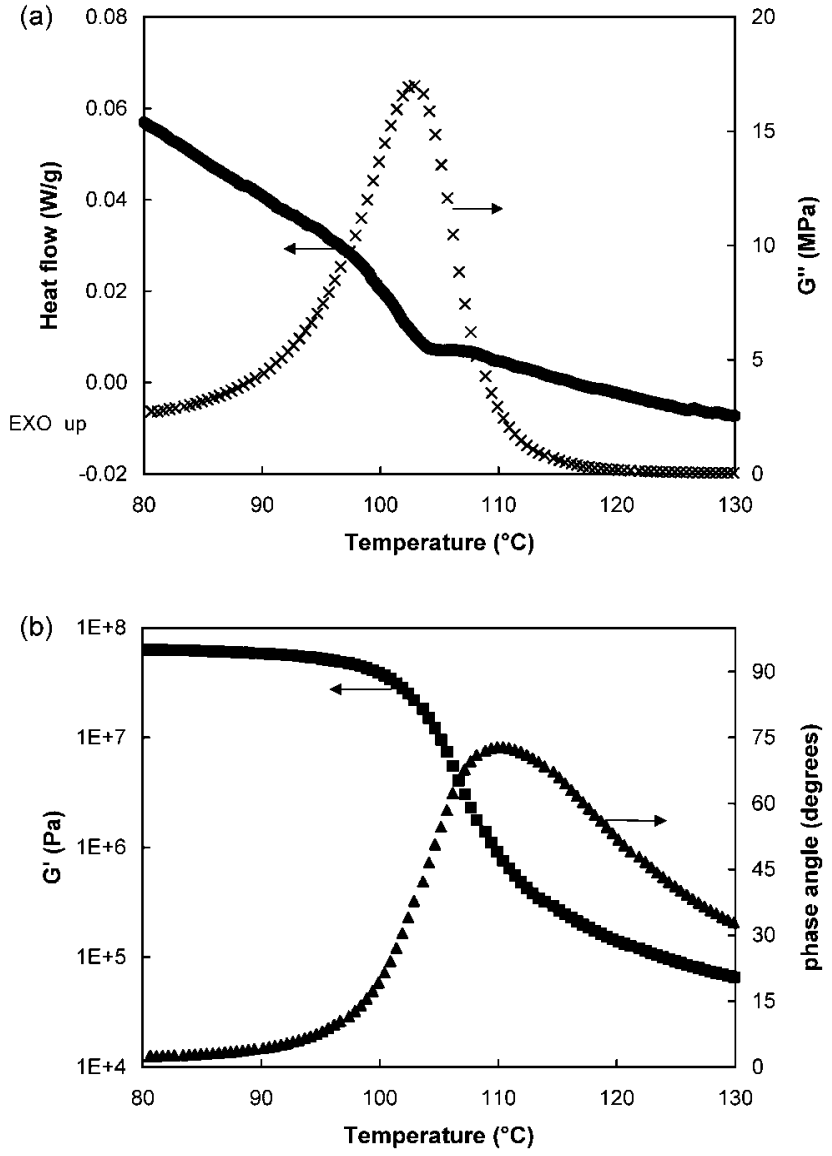

FIG. 4. Validation of the heat flow signal and corresponding rheological signals for the glass transition of polystyrene measured at a rate of $2.5^{\circ} \mathrm{C} \mathrm{min}^{-1}$. (a) Heat flow (line) and loss modulus $(\times)$; (b) storage modulus $(\boldsymbol{\square})$ and phase angle $(\mathbf{\Delta})$. All signals recorded using the RheoDSC setup.

is worse than a regular TA Instruments Q1000 DSC, but comparable to a TA Instruments 2920 DSC. Despite the immense changes applied to the original DSC cell environment, a reasonably stable and reproducible baseline could be obtained.

Following the modification process, the DSC cell was calibrated using a T4 calibration procedure. ${ }^{8}$ Subsequently, the heat flow signal was validated by measuring the glass transition of polystyrene (PS) $\left(M_{n}=140 \mathrm{~kg} \mathrm{~mol}^{-1}\right.$, $M_{w}=230 \mathrm{~kg} \mathrm{~mol}^{-1}$, Sigma-Aldrich) upon heating at $2.5^{\circ} \mathrm{C} \mathrm{min}-1$ (without modulation). It can be seen that the glass transition is well resolved in both heat flow and loss modulus $G^{\prime \prime}$ [Fig. 4(a)]. Furthermore, the glass transition temperature $\left(104^{\circ} \mathrm{C}\right)$ and the magnitude of the heat capacity change at $T_{g}$ calculated from the heat flow change at $T_{g}$ $\left(0.33 \mathrm{~J} \mathrm{~g}^{-1} \mathrm{~K}^{-1}\right)$ are in good agreement with literature values $\left(103{ }^{\circ} \mathrm{C}\right.$ and $\left.0.30 \mathrm{~J} \mathrm{~g}^{-1} \mathrm{~K}^{-1}\right){ }^{11}$ For a further optimization of the heat flow signal and the baseline, the Tzero ${ }^{\mathrm{TM}}$ extended heat flow equation could be modified to model the new thermal environment of the calorimeter more precisely.

Figure 4(b) gives the evolution of other rheological signals for the glass transition of PS. As the temperature is increased, $G^{\prime \prime}$ decreases, and the material changes from an energy-elastic solid with a loss angle of a few degrees (well below $T_{g}$ ), to a viscoelastic material with a phase angle of about $30^{\circ}$ (above $T_{g}$ ). At the glass transition, $G^{\prime \prime}$ and $\delta$ go through a maximum.

It is worth noting that the highest storage modulus $G^{\prime}$ measured (close to $100 \mathrm{MPa}$ ) is limited by the compliance of the setup. Assuming that the instrument is a spring in series with the viscoelastic sample, the torsional stiffness measured in oscillation is a summation of the inverse of the torsional stiffnesses of the tool and sample (or a summation of their compliances), ${ }^{12}$

$$
\frac{1}{K_{\text {meas }}^{*}}=\frac{1}{K_{s}^{*}}+\frac{1}{K_{t}},
$$

where $K_{\text {meas }}^{*}$ is the measured complex torsional stiffness, $K_{s}^{*}$ is the actual complex sample torsional stiffness, and $K_{t}$ is the (elastic) torsional stiffness of the tool. The torsional stiffness $K$ of a cylindrical sample is defined as follows:

$$
K=G \frac{\pi}{2} \frac{R^{4}}{h}
$$

where $G$ is the shear modulus of the material in torsion, $h$ is the gap between the platens, and $R$ is the radius of the platens. The fourth order dependence of $K_{s}$ on the sample (rotor) radius explains why higher moduli can be measured (without correction) using smaller radius platens.

For the RheoDSC setup, the tool stiffness contains both contributions of the rotor and the stator (the fixation of DSC pan). To measure $K_{t}$, first the rotor was fixed to the crucible of the rheoDSC using a very thin $(h<5 \mu \mathrm{m})$ layer of a cyanoacrylate glue. Then the stiffness was determined by recording the displacement (in rad) for an oscillatory torque sweep from 500 to $5000 \mu \mathrm{N} \mathrm{m}$ (a range representative for measuring moduli in the order of $1 \mathrm{MPa}$ and above) at a $2 \mathrm{rad} \mathrm{s}^{-1}$ angular frequency. The resulting $K_{t}$ equals $3.0 \pm 0.2 \mathrm{~N} \mathrm{~m} \mathrm{rad}^{-1}$, with a phase angle of $1.9^{\circ}(0.03 \mathrm{rad})$. This implies that $1 \mathrm{~mm}$ thick samples with a modulus up to $8 \mathrm{MPa}$ can be measured with less than $10 \%$ deviation. Using the experimental $K_{t}$, the measured modulus can be corrected using

$$
\frac{1}{K_{s}^{*}}=\frac{1}{K_{\text {meas }}^{*}}-\frac{1}{K_{t}} .
$$

Note that for viscoelastic samples, the complex inversion needs to be made. For polystyrene sample having at $80^{\circ} \mathrm{C}$ a measured $G^{\prime}$ of $72.6 \mathrm{MPa}$ and $G^{\prime \prime}$ of $3.6 \mathrm{MPa}$, corrected values of 2.0 and $1.4 \mathrm{GPa}$, respectively, are obtained, which are of a more reasonable order of magnitude for a polystyrene about $20^{\circ} \mathrm{C}$ below the glass transition. It should be noted that as the stiffness of the sample increases, its contribution to the observed displacement decreases, and the determination of $K_{s}$ becomes less reliable: for a $1 \mathrm{~mm}$ thick sample the estimated error on $K_{s}$ reaches $50 \%$ for a modulus of about $0.3 \mathrm{GPa}$.

\section{CASE STUDIES}

The potential of combined measurements using the RheoDSC will be highlighted by two case studies: the iso- 
thermal cure of a thermosetting polymer and the flowinduced crystallization behavior of a thermoplastic polymer.

\section{A. Isothermal cure of a thermosetting system}

The RheoDSC was used to study the cure of diglycidylether of bisphenol A (DGEBA) (EPON 825, Shell) with methylenedianiline (MDA) (Sigma Aldrich). DGEBA-MDA was chosen as a model system because it is commonly used for fiber-reinforced composites, and because of its nonvolatile character, which is important since evaporation of reagents from the open crucible needs to be avoided. Stoichiometric mixtures of DGEBA-MDA were prepared by mechanically stirring weighed amounts of the materials at $80^{\circ} \mathrm{C}$ for $5 \mathrm{~min}$. Subsequently, the sample was loaded into the DSC crucible in the RheoDSC at $40{ }^{\circ} \mathrm{C}$, a temperature at which the reaction rate is sufficiently low. After bringing the rotor into contact with the sample, the temperature was raised to $80{ }^{\circ} \mathrm{C}$ at a rate of $30{ }^{\circ} \mathrm{C} \mathrm{min}^{-1}$ and then kept isothermal for $300 \mathrm{~min}$. The calorimetric and rheological signals were recorded in a combined MTDSC measurement, using an amplitude of $0.5^{\circ} \mathrm{C}$ and a $60 \mathrm{~s}$ period, and small angle oscillatory shear measurement, respectively. For the latter, the oscillation torque was initially set to reach a strain of about 3\% for an angular frequency of $2 \mathrm{rad} \mathrm{s}^{-1}$. As the reaction proceeds, each time the strain decreased below $0.5 \%$, the oscillation torque was automatically increased by a factor of 2, thus maintaining the strain between about $1 \%$ and $0.5 \%$.

In Fig. 5(a), the variation of the heat flow and heat capacity during the isothermal cure as recorded by MTDSC measurement in the RheoDSC is compared to the results obtained in a regular TA Instruments 2920 DSC. In the heat flow signal, a reaction exotherm of $360 \mathrm{~J} \mathrm{~g}^{-1}$ is observed, going through a maximum after $82 \mathrm{~min}$ due to the autocatalytic effect of hydroxyl groups formed during the reaction. ${ }^{13}$ In the heat capacity signal, a stepwise decrease in heat capacity of about $0.5 \mathrm{~J} \mathrm{~g}^{-1} \mathrm{~K}^{-1}$ due to reaction-induced vitrification is noted. ${ }^{7,14}$ As the cure temperature $\left(80^{\circ} \mathrm{C}\right)$ is well below the glass transition temperature at full conversion [approximately $175{ }^{\circ} \mathrm{C}$, (Ref. 15)], a transition from a rubbery or mobile to a glassy state occurs along the cure path., ${ }^{74-16}$ As a result, the epoxy-amine step-growth polymerization becomes diffusion controlled, resulting in a retardation of the cure kinetics. ${ }^{16}$

Comparing the calorimetric signals obtained by both instruments, the maxima in the heat flow and the remainder of the reaction exotherm are in good agreement, as is the stepwise decrease in heat capacity. However, the evolution of the reaction conversion cannot be calculated quantitatively from heat flow signal of the RheoDSC because of a slow stabilization of the heat flow signal after heating to the cure temperature at $30{ }^{\circ} \mathrm{C} \mathrm{min}^{-1}$. This can be improved by reducing the difference in thermal lag between sample and reference side using a slower heating rate or balancing the heat capacities. Another option is to subtract a baseline acquired using the same temperature program with a fully cured material. Similar approaches are used in conventional DSC experiments (e.g., for studying a fast isothermal reaction or crystallization).

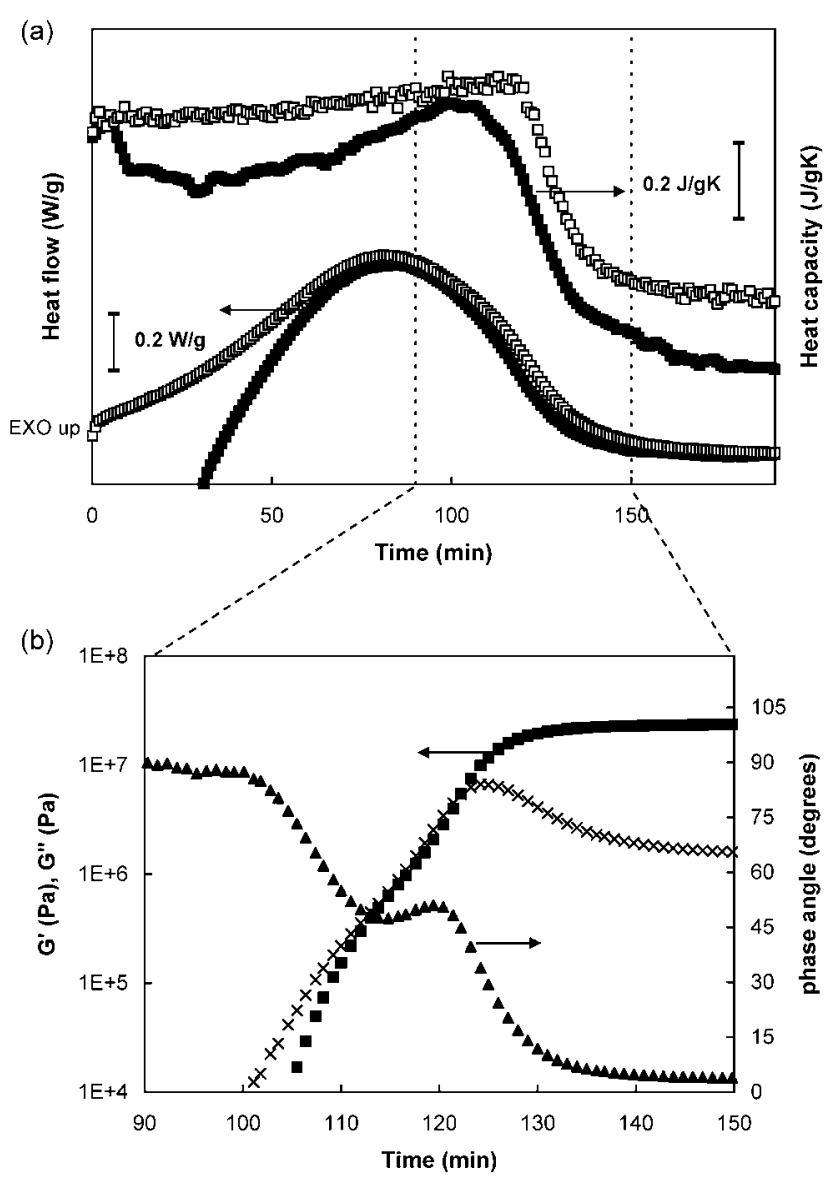

FIG. 5. RheoDSC measurement of the cure of a stoichiometric DGEBAMDA thermosetting system at $80^{\circ} \mathrm{C}$ : (a) Heat flow and heat capacity for the RheoDSC (ם) and for a regular TA Instruments 2920 DSC ( $\square$ ); (b) storage modulus $(\boldsymbol{\square})$, loss modulus $(\times)$, and phase angle $(\boldsymbol{\Delta})$ measured simultaneously in the RheoDSC.

The rheological signals measured show the large changes in the viscoelastic behavior of the cross-linking epoxy-amine system [Fig. 5(b)]. In the early stages of the reaction, the reactive mixture is a low viscosity liquid with a viscosity of about $0.05 \mathrm{~Pa} \mathrm{~s}$ (as measured at $80{ }^{\circ} \mathrm{C}$ in steady shear with $4 \mathrm{~cm}$ platens). The initial viscosity measured in the RheoDSC experiment is 200-300 Pa s and the loss angle about $90^{\circ}$. Based on the minimum torque specifications of the instrument $(0.1 \mu \mathrm{N} \mathrm{m})$, the rotor diameter $(4.5 \mathrm{~mm})$, and the measurement conditions used, the theoretical lower limit of the viscosity measurement range of the RheoDSC is about $100 \mathrm{~Pa}$ s (using steady shear measurements, viscosities down to $1 \mathrm{~Pa} \mathrm{~s}$ can be measured). After curing for $90 \mathrm{~min}$, the viscosity increases above $300 \mathrm{~Pa}$ s and significant values are obtained. With the progress of the cure reaction, $G^{\prime}$ and $G^{\prime \prime}$ increase rapidly, while the loss angle decreases as the molecular weight of the growing chains increases and the sample gradually becomes more elastic. When an incipient network is formed, flow becomes impossible, and the material transforms from a viscous to a rubbery state. This transformation, called "gelation," is often characterized by the crossover of $G^{\prime}$ and $G^{\prime \prime}$ (observed after $122 \mathrm{~min}$ at 5.4 MPa), although more refined methods exist. ${ }^{17}$ Note that this tech- 
nologically important transition cannot be observed in the calorimetric signals.

As the cure reaction continues, the $T_{g}$ ultimately reaches the cure temperature, and vitrification occurs. The transition to a glassy state should be observed as an increase in storage modulus, while the loss modulus goes through a maximum [at 124 min, Fig. 5(b)]. The rheological results indicate that gelation and vitrification closely follow each other. This is in agreement with the limited difference between the estimated degrees of conversion at gelation and vitrification of $58 \%$ and 77\%, respectively. ${ }^{15}$ A closer look at Figs. 5(a) and 5(b) shows that vitrification as observed in the heat capacity $\left(t_{1 / 2 \triangle C p}=129 \mathrm{~min}\right)$ occurs slightly later than the maximum in $G^{\prime \prime}(124 \mathrm{~min})$ recorded in the same experiment. As the frequency of the imposed perturbation for rheometry $(1 \mathrm{~Hz})$ is higher than for MTDSC $(1 / 60 \mathrm{~Hz})$, vitrification as characterized by means of $G^{\prime \prime}$ is expected at slightly lower conversion and shorter reaction time. ${ }^{18}$

It should be noted that the evolution of the moduli during vitrification and the final measured storage modulus (24 MPa) of the isothermally cured glassy epoxy-amine network is again limited by the compliance of the setup used, as discussed in Sec. III. In addition to the isothermal monitoring of the reaction, RheoDSC also allows to measure glass transitions of the (partially) cured systems under nonisothermal conditions. After a reaction time of $300 \mathrm{~min}$, the partially cured thermoset was first cooled and subsequently heated to $200{ }^{\circ} \mathrm{C}$ to achieve full conversion. The $T_{g}$ of the fully cured thermoset was $174{ }^{\circ} \mathrm{C}$, in good agreement with the reported value of $175^{\circ} \mathrm{C}$. ${ }^{15}$

\section{B. Flow-induced crystallization}

During the processing of semicrystalline thermoplastics, polymer melts are often subjected to severe flow conditions prior or even during their solidification resulting from polymer crystallization. Moreover, flow is known to modify the dynamics of polymer melts and to alter their crystallization kinetics. ${ }^{19-22}$ As shear can be imposed, the hyphenated RheoDSC method offers unique opportunities for the investigation of flow-induced crystallization processes. Indeed, in situ monitoring of crystallization is not possible using a traditional DSC, since no flow field can be applied. Furthermore, when studying the crystallization under quiescent conditions, experimental data from rheometry and calorimetry cannot easily be confronted with each other due to important differences in temperature control and sample volumes. The newly developed RheoDSC device is not restrained by these limitations: both the application of the flow field and the measurement of the calorimetric signal are performed in one single instrument.

As a model system for this flow-induced crystallization study, syndiotactic polypropylene was selected (sPP, $M_{n}$ $=63 \mathrm{~kg} \mathrm{~mol}^{-1}, M_{w}=250 \mathrm{~kg} \mathrm{~mol}^{-1}$, stereospecificity approximately $80 \%$, Fina Research). The samples were premelted in the RheoDSC to ensure good contact between sample and crucible. The rotor was then brought in contact with the molten sample. Prior to each crystallization experiment, the sample was held at $180{ }^{\circ} \mathrm{C}$ for $5 \mathrm{~min}$ to erase its thermal history, after which it was subjected to a slow cooling at

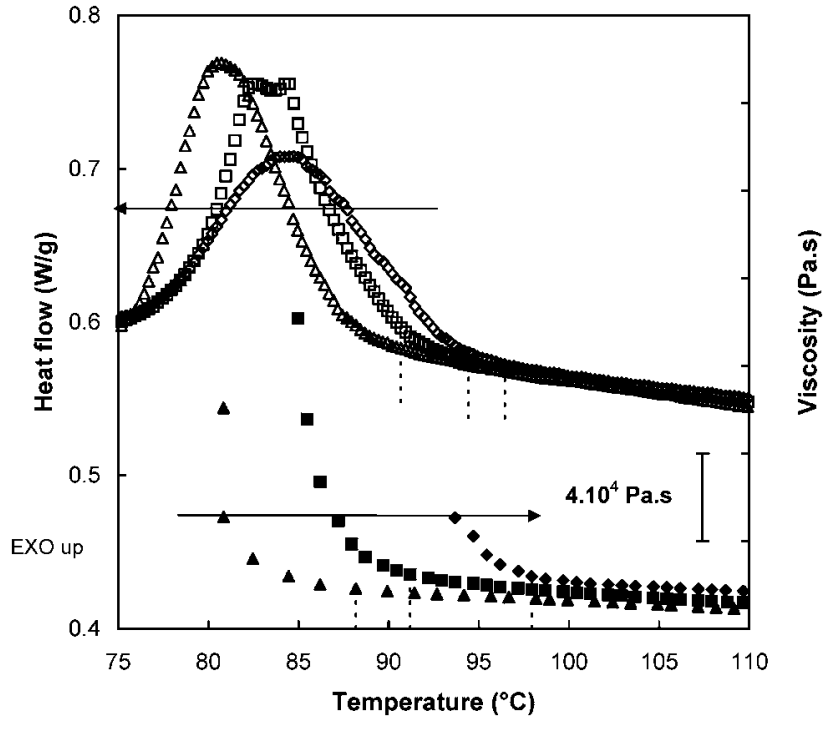

FIG. 6. RheoDSC measurement of the nonisothermal crystallization of syndiotactic polypropylene during cooling at $2.5^{\circ} \mathrm{C} \mathrm{min}^{-1}$ under different shear conditions: Heat flow (open symbols) and viscosity (filled symbols) for quiescent conditions $(\mathbf{\Delta} \triangle)$ and at shear rates of $0.2 \mathrm{~s}^{-1}(\square \square)$ and $0.8 \mathrm{~s}^{-1}$ $(\diamond \diamond)$. The dashed lines indicate the first points with a significant deviation from the baseline.

$2.5^{\circ} \mathrm{C} \min ^{-1}$ to room temperature and subsequently reheated under the same conditions. For the quiescent experiment, the rheology was monitored in oscillation mode using an angular frequency of $2 \mathrm{rad} \mathrm{s}^{-1}$ and a strain of $5 \%$. For the crystallization experiments under flow, the samples were sheared at shear rates of 0.2 and $0.8 \mathrm{~s}^{-1}$ during the entire cooling segment.

Figure 6 combines the rheometric and calorimetric signals for crystallization in quiescent conditions and under flow at different shear rates. In the heat flow the crystallization exotherm is observed, while the increase in the (real component of the) viscosity can be attributed to the formation of the crystalline structure. Even at a moderate shear rate of $0.2 \mathrm{~s}^{-1}$, crystallization starts several degrees higher as compared to quiescent conditions. Moreover, under quiescent conditions and at $0.2 \mathrm{~s}^{-1}$, crystallization is observed in the calorimetric signals before being observed in the rheological signals. Taking as the start of crystallization the temperature at which the first significant deviation from the baseline is observed, the difference is about $3{ }^{\circ} \mathrm{C}$. At $0.8 \mathrm{~s}^{-1}$, the first significant deviation from the baseline is first observed in the viscosity, and $1.5^{\circ} \mathrm{C}$ later in the heat flow.

Although these are preliminary measurements using a prototype instrument, the observed behavior might be explained by the specific morphology developed during crystallization. In the absence of a steady shear flow, the crystallites need to attain a proportionally larger size in order to influence the viscoelastic properties to a detectable extent. When a shear flow is applied, a higher nucleation density is achieved, resulting in a finer crystalline morphology. ${ }^{22,23} \mathrm{At}$ sufficiently high shear rates, this might cause a proportionally much more significant change in the viscoelastic properties compared to the situation under quiescent conditions and may therefore lead to a significant increase in viscosity 
earlier in the crystallization process. For the heat flow measurement, the rate of volumetric growth of crystalline lamellae may be more important than the number of crystal nuclei the crystals started from. To confirm these assumptions, a more thorough investigation of the morphology development under shear and its influence on the viscoelastic and calorimetric properties is required.

\section{CONCLUSIONS}

By combining a rheometer with a DSC, the simultaneous measurement of calorimetric and rheological signals on 10-30 mg samples was realized. The signals were reliable and of good quality, without largely affecting the performance provided by the two stand-alone techniques. By performing the measurements simultaneously on a single sample undergoing an in situ imposed flow and temperature history, complementary information is obtained in a quantitative way. For crystallizing systems, the rheological evolution can be directly linked to the advancing crystallization and crystallinity as detected in the calorimetric signals. For reacting systems the evolution in the viscosity and the occurrence of gelation and vitrification as observed from the rheological signals can be directly related to the advancing reaction conversion derived from the calorimetric data. The examples discussed show the wide applicability of this novel technique and demonstrate the added value of a hybrid RheoDSC setup for the characterization of material systems with complex chemorheological changes and for studying transformations that are influenced by the flow conditions.

\section{ACKNOWLEDGMENTS}

This work was supported by the Research FoundationFlanders (FWO) and by TA Instruments.
${ }^{1}$ R. H. Somani, B. S. Hsiao, A. Nogales, H. Fruitwala, S. Srinivas, and A. H. Tsou, Macromolecules 34, 5902 (2001).

${ }^{2}$ R. H. Somani, B. S. Hsiao, A. Nogales, S. Srinivas, A. H. Tsou, I. Sics, F. J. Balta-Calleja, and T. A. Ezquerra, Macromolecules 33, 9385 (2000).

${ }^{3}$ J. A. Martins, W. Zhang, and A. M. Brito, Rev. Sci. Instrum. 76, 105105 (2005).

${ }^{4}$ R. F. Garritano, M. Padmanabhan, and M. Goncharko, European Patent No. EP0750186-A3 (1998); M. Padmanabhan, Japanese Patent No. JP9101278 (1997); R. F. Garritano, M. Goncharko, and M. Padmanabhan, U.S. Patent No. 5520042 (1996).

${ }^{5}$ W. Nagatake, T. Takahashi, Y. Masubuchi, J.-I. Takimoto, and K. Koyama, Polymer 41, 523 (2000).

${ }^{6}$ M. Reading, A. Luget, and R. Wilson, Thermochim. Acta 238, 295 (1994).

${ }^{7}$ Modulated-Temperature Differential Scanning Calorimetry: Theoretical and Practical Applications in Polymer Characterisation (Hot Topics in Thermal Analysis and Calorimetry), edited by M. Reading and D. J. Hourston (Springer, London, 2006).

${ }^{8}$ R. L. Danley, Thermochim. Acta 395, 201 (2003).

${ }^{9}$ R. L. Danley, Thermochim. Acta 402, 91 (2003).

${ }^{10}$ G. Mosselmans, M. Biesemans, R. Willem, J. Wastiels, M. Leermakers, H. Rahier, S. Brughmans, and B. Van Mele, J. Therm Anal. Calorim. 88, 723 (2007)

${ }^{11}$ E. Marti, E. Kaisersberger, and E. Moukhina, J. Therm Anal. Calorim. 85, 505 (2006).

${ }^{12}$ K. Schröter, S. A. Hutcheson, X. Shi, A. Mandanici, and G. B. McKenna, J. Chem. Phys. 125, 214507 (2006).

${ }^{13}$ B. A. Rozenberg, Adv. Polym. Sci. 75, 113 (1986).

${ }^{14}$ G. Van Assche, A. Van Hemelrijk, H. Rahier, and B. Van Mele, Thermochim. Acta 268, 121 (1995).

${ }^{15}$ S. Swier, G. Van Assche, and B. Van Mele, J. Appl. Polym. Sci. 91, 2814 (2004).

${ }^{16}$ G. Wisanrakkit and J. K. Gillham, J. Coat. Technol. 62, 35 (1990); M. T. Aronhime and J. K. Gillham, Adv. Polym. Sci. 78, 83 (1986).

${ }^{17}$ H. H. Winter and F. Chambon, J. Rheol. 30, 367 (1986); F. Chambon and H. H. Winter, J. Rheol. 31, 683 (1987).

${ }^{18}$ G. Van Assche, B. Van Mele, and Y. Saruyama, Thermochim. Acta 377, 1 (2001).

${ }^{19}$ P. Supaphol and W. Harnsiri, J. Appl. Polym. Sci. 100, 4515 (2006).

${ }^{20}$ R. M. Khafagy, J. Polym. Sci., Part B: Polym. Phys. 44, 2173 (2006).

${ }^{21}$ C. De Rosa and F. Auriemma, Prog. Polym. Sci. 31, 145 (2006).

${ }^{22}$ P. Supaphol, P. Thanomkiat, and R. A. Phillips, Polym. Test. 23, 881 (2004).

${ }^{23}$ F. Jay, J. M. Haudin, and B. Monasse, J. Mater. Sci. 34, 2089 (1999). 